\title{
Welcome to Surgeries: A New Open Access Platform for Clinical and Experimental Research and Developments in All Fields of Surgery
}

\author{
Cornelis F.M. Sier \\ Department of Surgery, Leiden University Medical Centre, 2333 ZA Leiden, The Netherlands; c.f.m.sier@lumc.nl \\ Received: 3 March 2020; Accepted: 3 March 2020; Published: 5 March 2020

Dear readers, authors, and reviewers of Surgeries,

Because of rapidly evolving techniques and novel insights, the singular word -surgery-, derived from the ancient Greek $\chi \varepsilon\llcorner\rho o v \rho \gamma \iota \alpha$, meaning hand labor, can hardly comprise the full plethora of topics, procedures, and techniques within the field of surgery. Recent state-of-the-art technical innovations like robotic and image-guided surgery, and novel concepts like Watch and Wait strategies for cancer treatment seem only the top of the iceberg of future developments. Therefore, we would like to welcome you to the novel open access journal Surgeries with an s, as in plural.

Despite the existence of some excellent surgery-related journals and the regular introduction of novel open access attempts, we are convinced there is room for Surgeries. Probably the best reason being, that from the 203 surgery-related journals indexed in Web of Science, only 11 are indicated to be full open access. The key advantage of open access is obviously global availability to the readers, but this is only valid as long as the quality of the peer reviewing is guaranteed. Surgeries will pursue to become a high-quality open access journal for original clinical, theoretical, and experimental advances and research in all fields of surgery. We will publish articles, communications, and reviews, covering basic, translational, and clinical studies and concepts in basically all surgical disciplines. Obviously, in a time where "interdisciplinary" is written in capitals, we will especially welcome contributions integrating developments from other scientific disciplines, like artificial intelligence, into surgery.

Surgeries outstanding international and interdisciplinary editorial board, consisting of surgeons and scientists, together with a highly effective editorial office team, are determined to offer our colleagues an open access platform, rather than just another journal about surgery. Next to their role in providing a rapid but high-quality review procedure, the editors will actively contribute by organizing special issues focused on topics of high interest. Together and with the help of authors and reviewers, we aim to develop Surgeries into an indispensable, high quality, and easily accessible source of information for surgery-related professionals and beyond.

(C) 2020 by the author. Licensee MDPI, Basel, Switzerland. This article is an open access article distributed under the terms and conditions of the Creative Commons Attribution (CC BY) license (http://creativecommons.org/licenses/by/4.0/). 